\title{
Development of a blocking logic checking apparatus for substation measurement and control devices
}

\author{
Li Chen, Bingwei Gao, Feng Qi, Bifu Qian, and Lei Zhang \\ Wenzhou Power Supply Company of SGCC, 325000 Zhejiang Wenzhou, China
}

\begin{abstract}
After transforming substation measurement and control devices, the traditional method of blocking logic check needs to go back and forth among the measurement and control devices, and repeatedly connect a large number of wires, which lead to tedious operation and low efficiency. This paper developed a logic verification device based on $4 G$ wireless communication, which used the Modbus/TCP transport protocol to access Ethernet, to complete the real-time data communication with the mobile control terminal. And this paper made a request to the logic controller according to practicality and cost. And the terminal software interface was designed by the orthogonal experimental design idea. The development of this device provided a new and efficient method for logic verification.
\end{abstract}

\section{Introduction}

Maloperation prevention is an important item in substation operation management. In maloperation accident, it not only hurts operators severely, but also damages operating equipment seriously, even causes blackout [1].

When dispatching department and supervising system control the measurement and control device(MACD) remotely, MACD analyzes the maloperation prevention blocking(MPB) logic by acquired information and interlock information about other MACDs. If the condition is fulfilled, the contact will be closed, which means can be controlled remotely. Otherwise, it blocks operation.

Checking the correctness of bay level blocking logic is a considerable item for new MACD acceptance [2]. Traditional process of checking blocking logic demands that changing wires back and forth among interlocking MACDs for satisfying different requirements of tele-signaling input, as the blocking method is calculated by MACD with the state of contacts of field equipment [3]. It not only costs energies and time, but also likely causes "repeat checking" and "omit checking", and leads to potential risk. Therefore, this paper developed a blocking logic checking apparatus of substation MACD by using $4 \mathrm{G}$ communication technology. And it offered a new and efficient method for logic verification to engineers.

\section{The principle of device}

\subsection{The interlocking principle of bays}

The MPB of MACD consists of block in a bay and interlock among bays. The block in a bay is simple because information library of equipment is in one MACD. The interlock among bays is much more complex as data is derived from other bays besides local data [4]. The classic flow of blocking logic judgement is shown in Figure 1.

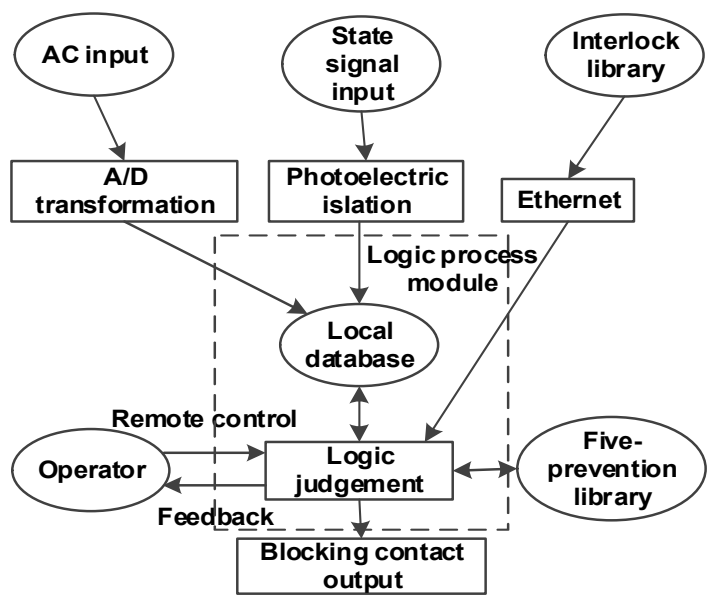

Fig. 1. The implementation process of blocking logic 


\subsection{The origin of the question}

Traditional blocking logic verification consists of five segments according to the above principle. First, completing security precautions. Second, connecting and disconnecting circuits. Third, going back and forth among MACDs. Fourth, confirming input signal. Last, testing the tele-controlling.

Figure 2 is showing a part of primary electric wiring diagram in a $220 \mathrm{kV}$ substation. It contains three bays: Wenjiang 2391 Line, Wennan 2392 Line and bus coupler.

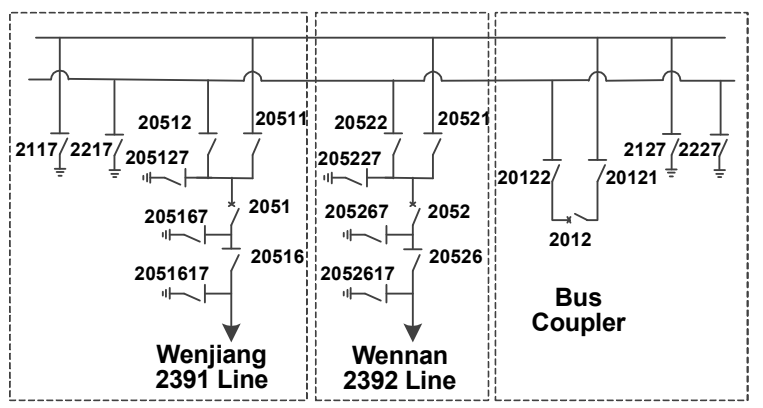

Fig. 2. The wiring diagram of multi-bay in a $220 \mathrm{kV}$ substation

For instance, according to requirement of MPB [5], the blocking logical expression of operating No.20512 disconnector of Wenjiang 2391 Line is showed below.

$F_{C L s}(20512)=2012(1) \& 20121(1) \& 20122(1) \&$

20511(1) \& 2117(0) \& 2217(0) \& 2127(0) \& 2227(0)

OR 20511(0) \& 2051(0) \& 205127(0) \& 205167(0) \&

2217(0) \& 2227(0)

Where $F_{C L s}$ means consequence of blocking logic, and (0) means open, and (1) means close.

According to above expression, when Wenjiang 2391 Line is switching bus, double bus should be in parallel operation, which means bus coupler(2012), I-bus disconnector(20122) of bus coupler and II-bus disconnector(20121) of bus coupler are close, besides the blocking of this line. Thus, to verify the blocking logic of No.20512 disconnector, engineers have to walk back and forth frequently among MACD of Wenjiang 2391 Line, MACD of bus bar and MACD of bus coupler for changing circuits in traditional method. Therefore, it will promote efficiency of verification greatly if there is a way that taking the place of changing circuits manually, and getting feedback of input in time.

\subsection{Logic verification scheme based on $4 \mathrm{G}$ communication technology}

4G technology has the advantages of no distance limitation and high quality transmission. Based on these advantages, this paper used 4G communication technology and developed a blocking logic controller with cross-platform control software, which achieved wireless verification of blocking logic of MACDs, and solved the low-efficiency problem caused by frequent changing circuits in the traditional method.
While logic verification of one MACD involved blocking information of other bays, this paper had to configure blocking logic controller in this MACD. Every controller had 16-channel contacts, and these contacts could be connected to tele-signaling circuit of MACD. The controller drove corresponding electric relays after that engineers issued tele-controlling orders. Then, relays changed the state of contacts for meeting the demand about blocking input.

\section{The design of logic controller}

\subsection{The design of circuitry}

Considering the utility of controller, economy and substation circumstances, the goals of the controller is shown below.

(1) Power-supply Module: For the convenience of engineering application, this paper chose Li-on battery as power supply because of small size and good heat dissipation. Its voltage output is $3 \mathrm{~V}-24 \mathrm{~V}$, and can be used for ten days without charging.

(2) Electric Relay Module: Considering the electromagnet interference of substation, this paper selected AQW214S relay as low drive current, high voltage-endurance and low price.

(3) Communication Interface Module: Adopting M6310 4G communication module. It embeds entire $\mathrm{TCP} / \mathrm{IP}$ protocol, and is antijamming, without distance limitation.

(4) Process Unit Module: This paper chose STM32F series MCU as the process unit. Its work frequency is $0 \sim 35 \mathrm{MHz}$, and it could meet the requirement of the speed of transmitting TCP/IP data packets. Besides, it has low power consumption and low price.

(5) Other Modules: To avoid data loss while power cut, this paper selected EEPROM as memory chip.

The entire structure of the device is shown in Figure 3.

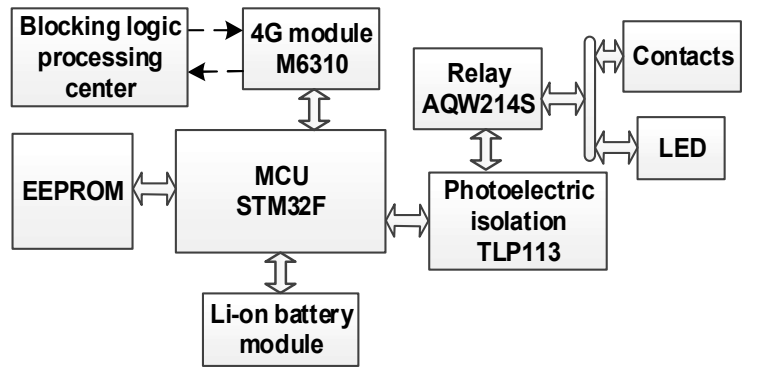

Fig. 3. The diagram of the hardware circuit

\subsection{The design of communication interface}

The logic controller based on standard Modbus/TCP protocol utilized Ethernet to achieve data interchange between logic controller and server by reliable peer-to-peer communication built on TCP protocol. Data frame received by M6310 module will be checked 
whether matching native IP address automatically. If the data packet was valid, it would be sent to buffer. And MCU would complete the analysis of tele-controlling order and the feedback of blocking contact. This process was crucial for communication between logic controller and system. The procedure of data interchange is shown in following diagram.

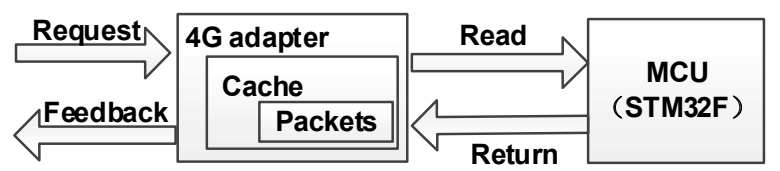

Fig. 4. The diagram of data interaction

\section{The design of terminal application}

\subsection{The design of function interface}

The development of this device aimed at simplifying the flow of logic verification, and promoting efficiency of checking. Therefore, this paper focused on utility and operability when designing function interface of the client. There were four major factors influencing operating efficiency, considering reality of logic verification.

(1) Association Mode of Channel Contacts

(2) Control Type of Contacts

(3) The Number of Local Control Units

(4) The Number of Channels

This paper chose an optimum combination through experiments. Parts of the function interface are shown in Figure 5.

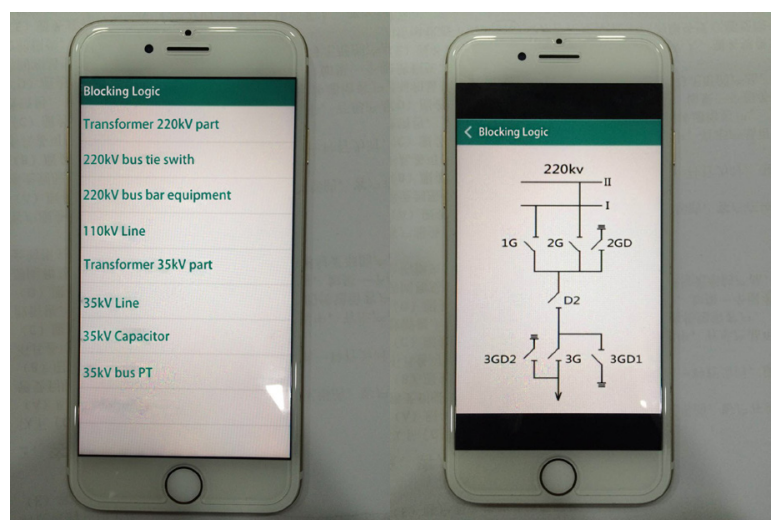

Fig. 5. The client function interface

\subsection{Mechanism of mutually exclusive logic}

The tele-signaling input usually indicates the state of on/off by dual position contact for most of MACDs. If a disconnector is close, the input of normal open contact is 1 , and the input of normal close contact is 0 . Similarly, when a disconnector is open, the signal of normally open contact and normal close contact is reverse. If one of contacts is adhesive or broken, the input will be either 1 or 0 . This case is classified as uncertain state by MACD, and it leads to deadlock of MACD. Thus, this paper had to take correctness of contact output into consideration when simulating tele-signaling input by the logic controller. This application utilized the mechanism of mutually exclusive logic on principal and subordinate channels to simplify the operation and avoid deadlock situation. That was, principal and subordinate channels were related to one contact of logic controller respectively. And while engineers gave a tele-controlling order by the mobile terminal, values of principal and subordinate channels were reverse automatically.

For example, to close No.20516 disconnector of $220 \mathrm{kV}$ Wenjiang Line shown in Figure 3, the data frame of tele-controlling order sending to controller was $0 \times 19$ $0 \times 56$. Converting it to binary number, and every two adjacent bits corresponded to control bit received from principal and subordinate channels ( 1 means close, 0 means open), except bit.14 and bit.15. The definition of control bits is shown in Table 1.

Table 1. The definition table of mutually exclusive logic

\begin{tabular}{|c|c|c|}
\hline Control Bit(bit0 bit13) & No. & Definition \\
\hline bit. $0=0$ & \multirow{2}{*}{20511} & \multirow{2}{*}{ Close } \\
\hline bit. $1=1$ & & \\
\hline bit. $2=1$ & \multirow{2}{*}{20512} & \multirow{2}{*}{ Open } \\
\hline bit. $3=0$ & & \\
\hline bit. $4=1$ & \multirow{2}{*}{205127} & \multirow{2}{*}{ Open } \\
\hline bit. $5=0$ & & \\
\hline bit. $6=1$ & \multirow{2}{*}{2051} & \multirow{2}{*}{ Open } \\
\hline bit. $7=0$ & & \\
\hline bit. $8=1$ & \multirow{2}{*}{205167} & \multirow{2}{*}{ Open } \\
\hline bit. $9=0$ & & \\
\hline bit. $10=0$ & \multirow{2}{*}{20516} & \multirow{2}{*}{ Close } \\
\hline bit. $11=1$ & & \\
\hline bit. $12=1$ & \multirow{2}{*}{2051617} & \multirow{2}{*}{ Open } \\
\hline bit. $13=0$ & & \\
\hline
\end{tabular}

Storage of logic controller reserved current state information about every contact, and MCU compared received control bit with data of relevant channels. If the result of comparison is consistent, it will not change the state of contact; otherwise, it changed according to control bit.

\section{Flow of verification}

To ensure the integrality of checking MPB, and simplify the procedure, this paper compiled the standard operation procedure and logic verification scheme of this device. The specific flow is shown in Figure 6. 


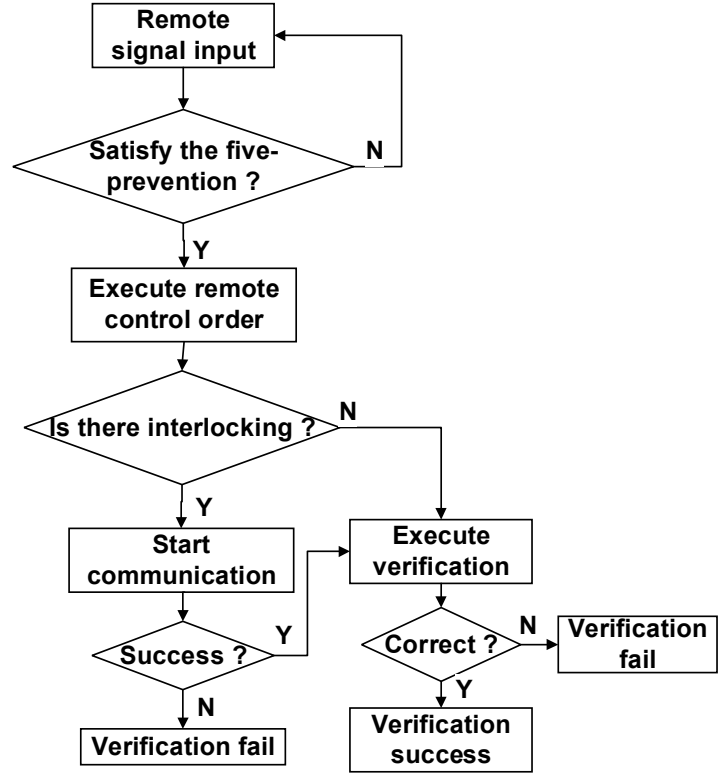

Fig. 6. Verification flow

(1) When checking a certain logic, this paper had to configure a logic controller in a relevant MACD first, and connected testing wires into tele-signaling input circuits.

(2) Controlling the state of contacts remotely through terminal application to meet the requirement of five-prevention. And this paper inspected it on supervising system or graphic interface of application.

(3) Giving a tele-controlling order by supervising system after completing the setting of contacts and meeting the demand of blocking logic. Once the MACD received instruction, it judged whether the equipment involved interlocking of other bays. If the interlock exited, it started interlocking communication first to acquire real time data; otherwise, it came to logic verification directly.

(4) The MACD computed MPB logic on the basis of local information and interlocking information of other bays, and sent the result of tele-controlling verification to the supervising system for testing the correctness of MPB logic.

(5) Judging the returned result to check the validity of MACD.

\section{Test of achievement}

In a retrofit project of MACD, this paper used traditional method and this device to verify the logic of the same bay, and wrote the time consumption down. The experimental data is shown in Table 2.

Comparing with the traditional method, as a result, wireless verification with this device promoted work efficiency greatly, and it assured the correctness and integrity of checking MPB.
Table 2. The test table of experiment

\begin{tabular}{|c|c|c|}
\hline Project & $\begin{array}{c}\text { 220kV Liao } \\
\text { 2Q94 Line }\end{array}$ & $\begin{array}{c}\text { 220kV 1\# } \\
\text { Transformer }\end{array}$ \\
\hline Model & CSI-200E & NSD500 \\
\hline Number of Trials & 5 & 3 \\
\hline Traditional Method / minute & 146.6 & 131 \\
\hline New Method / minute & 65.5 & 52.8 \\
\hline Logic Verification & Correct & Correct \\
\hline
\end{tabular}

\section{Conclusions}

The traditional method of checking MPB logic costs energies and time greatly. This paper provided a MPB logic verification device of MACD based on $4 \mathrm{G}$ communication. It's a new and efficient method for logic verification. Engineers could complete the verification through a mobile terminal. It promoted efficiency greatly. Meanwhile, it assured the safety of equipment. With the development of $4 \mathrm{G}$ and mobile devices, this method has favorable application prospect.

\section{References}

1. Y. Sun, L. Hou, P. Jie. Implementation of maloperation proof on platforms of relay and control units between bays. Automation of Electric Power Systems, 30, 81-85 (2006)

2. L. Zhang, B. Qian, X. Liu. Some Problems Analysis of Transformation of CSI-200EA Measurement and Control Equipment. Diangong Dianqi, 04, 35-37 (2015)

3. W. Zhou, L. Zhou. The Advantages and Disadvantages of Computer Monitoring and Control Device. Science \& Technology Information, 10, 123 (2012)

4. B. Duan, G. Chen, Y. Lin. On-Line Interlocking Implementation Based on Process Bus Communication of Digital Substation. Power System Technology, 19, 37-43 (2009)

5. Y. Gu, W. Pi, C. Yang. Application analysis of anti-mistake defense system in substation. RELAY, 02, 66-70 (2005) 